\title{
Development of a distance-independent wireless passive RF resonator sensor and a new telemetric measurement technique for wireless strain monitoring
}

\author{
Akbar Alipour ${ }^{a}$, Emre Unal ${ }^{a}$, Sayim Gokyar ${ }^{a}$, Hilmi Volkan Demir $^{\mathrm{a}, \mathrm{b}, *}$ \\ a Department of Electrical and Electronics Engineering, Department of Physics, and UNAM- National Nanotechnology Research Center and Institute of \\ Materials Science and Technology, Bilkent University, Bilkent, 06800, Ankara, Turkey \\ ${ }^{\mathrm{b}}$ LUMINOUS! Center of Excellence for Semiconductor Lighting and Displays, School of Electrical and Electronic Engineering, School of Mathematical and \\ Physical Sciences, Nanyang Technological University, Singapore 639798, Singapore
}

\section{A R T I C L E I N F O}

\section{Article history:}

Received 14 September 2016

Received in revised form

15 December 2016

Accepted 9 January 2017

Available online 11 January 2017

\section{Keywords:}

Strain sensor

Passive RF resonator

Inductive coupling

Wireless monitoring

\begin{abstract}
A B S T R A C T
We proposed and developed a novel wireless passive RF resonator scheme that enables telemetric strain sensing avoiding the need for calibration at different interrogation distances. The specific architecture of the proposed structure allows for strong inductive coupling and, thus, a higher wireless signal-to-noise ratio. Here, in operation, the frequency scan of the sensor impedance was used to measure simultaneously both the impedance amplitude and resonance frequency. Using this wireless sensor, we further introduced a new telemetric monitoring modality that employs full electrical characteristics of the system to achieve correct strain extraction at any interrogation distance. In principle, any deformation of the sensor structure results in the resonance frequency shift to track strain. However, changing of the interrogation distance also varies the inductive coupling between the sensor and its pick-up antenna at the interrogation distance. Therefore, at varying interrogation distances, it is not possible to attribute an individual resonance frequency value solely to an individual strain level, consequently resulting in discrepancies in the strain extraction if the interrogation distance is not kept fixed or distance-specific calibration is not used. In this work, we showed that by using both the proposed passive sensor structure and wireless measurement technique, strain can be successfully extracted independent of the interrogation distance for the first time. The experimental results indicate high sensitivity and linearity for the proposed system. These findings may open up new possibilities in applications with varying interrogation distance for mobile wireless sensing.
\end{abstract}

(C) 2017 Elsevier B.V. All rights reserved.

\section{Introduction}

Precise strain monitoring is essential in numerous strain sensing applications including implants, food quality control, and structural health monitoring [1-4]. Over the past few decades, enormous progress has been achieved in wireless passive and implantable strain monitoring techniques. Wireless readability, low cost, power source-free operation, and low perturbation from the surroundings are among the main advantages of the wireless passive approaches

\footnotetext{
* Corresponding author at: Department of Electrical and Electronics Engineering, Department of Physics, and UNAM- National Nanotechnology Research Center and Institute of Materials Science and Technology, Bilkent University, Bilkent, 06800, Ankara, Turkey.

E-mail address: volkan@bilkent.edu.tr (H.V. Demir).
}

over common active ones [5,6]. In telemetric strain sensing, the ability to wirelessly track the passive resonance is of critical importance [7]. The fundamental operating principle of wireless sensing results from inductive coupling between the passive radio frequency (RF) resonator and its reader antenna [8]. The concept of such passive RF resonators using different electromagnetic structures has been proposed and demonstrated for the purpose of wireless strain monitoring. Since the electromagnetic resonance frequency of a resonator is sensitive to its physical dimensions, any deformation in its geometry results in resonance frequency change [9].

The most recently studied architecture for wireless passive strain monitoring includes micro-strip patch antennas, radio frequency identification (RFID), and metamaterial-based RF resonators. The feasibility of using a circular micro-strip patch antenna (CMPA) was reported by Daliri et al. [10]. Using theoretical cal- 
culations and numerical simulations, the authors showed a linear relationship between the strain and the resonance frequency of the proposed system. Any change in the dimension of the CMPA structure changes the impedance of the system, which leads to the shift in resonance frequency. A complementary work on CMPAs was reported in [11]. It was shown that CMPAs can be used as a passive sensor for wireless strain monitoring in civil structures and aerospace. The effect of the conductivity of the host material on the wireless measurement efficiency was also investigated. It was demonstrated that strain can be monitored wirelessly in any desired direction using a linearly polarized horn antenna. However, due to the sensor signal interference with background reflections, only a limited interrogation distance was achieved.

RFID structures have also been investigated for wireless passive strain measurements. The RFID-based wireless sensors are fed by interrogation electromagnetic wave radiated from a reader antenna. Xiaohua et al. [12] developed a passive wireless antenna sensor for strain and crack detection. The antenna signal modulation was used to isolate the backscattered signal from the undesired environmental reflections.

From the previous works of our group, Melik et al. [13] proposed a highly sensitive metamaterial-based strain monitoring technique to track bone fracture healing. This sensor consists of double combshaped multiple split ring resonators (SRRs). The compact nested architecture of this SRR design with multiple gaps provides a lower operating frequency and higher sensitivity. In operation, the wireless passive metamaterial strain sensor is mounted on an implantable fracture fixation hardware to monitor and access the progression of bone fracture healing. When an external load is applied to the hardware, the strain is recorded remotely using the coaxial probe located in the proximity of the sensor. This sensor can measure strain based on the change in the capacitance of nested SRR that results in the transmission spectrum shift using distance-specific calibration. The in vivo results showed that the metamaterial-based passive strain sensor provides the ability to determine statistically important difference between the fracture healing and non-healing groups [14]. However, these sensors are resonant structures without a ground plane, so they exhibit relatively low signal-to-noise (SNR) ratio and quality factor. Our group also developed another metamaterial-based remote strain sensor system using an array of the SSRs patterned over a flexible Kapton gold clad substrate [15]. This approach was limited by the weak reflected signal from the sensor to the reader antenna.

In most of these studies, misalignment and variation in interrogation distance can change the transmission spectrum, consequently contributing some inaccuracy to strain extraction. To overcome this problem, in this paper, we propose a distanceindependent passive RF resonator sensor and a new telemetric measurement methodology for its wireless strain monitoring. Here the comb-shape split rings are patterned on both sides of the flexible dielectric to form a distributed capacitance and inductance tank circuit. The combination of the capacitance and inductance creates an LC resonator to operate at a certain frequency. The comb-shape split rings are aligned by $90^{\circ}$ rotation with respect to each other on both sides of the dielectric. This specific architecture of the sensor allows for the possible excitation of both of the layers by the same incoming electromagnetic wave. Thus, both of the layers contribute to the resonance frequency and the quality factor of the structure. We also introduce a new measurement technique of wireless strain sensing which, in combination with the proposed sensor, strain can be monitored independently of the interrogation distance. Based on this approach, possible discrepancies induced by the coupling coefficient variations are eliminated in the strain extraction. Experimental results indicate great linearity and sensitivity.

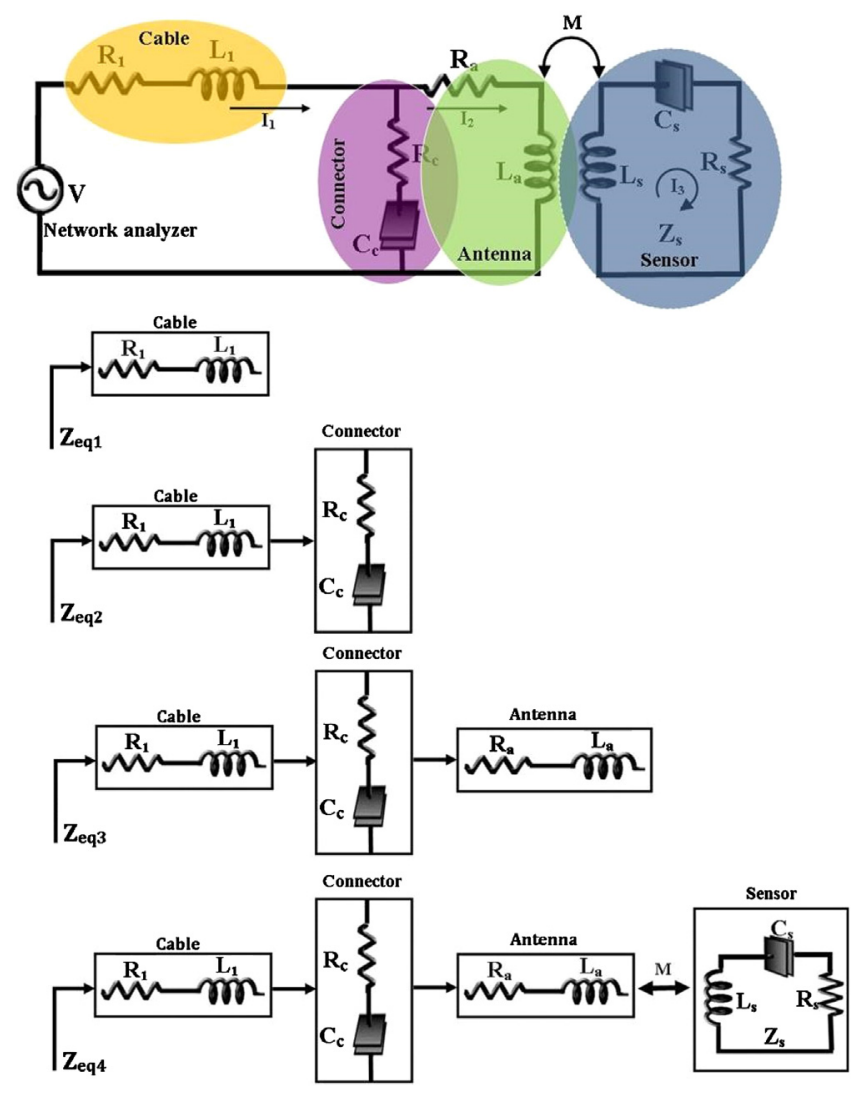

Fig. 1. Schematic of the proposed system. The sensor is inductively coupled to the pick-up reader antenna. To obtain the exact value of the resonance frequency $\left(f_{0}\right)$ of the sensor, all the resistance, capacitance and inductance values induced by the cable, the connector, and the antenna are included.

\section{Methods}

\subsection{Theory}

In our system, the principle of wireless strain monitoring based on inductive coupling is illustrated in Fig. 1. We include all the electrical components that come from the system (e.g., cable, connector, and antenna) to determine the accurate resonance frequency of the sensor. All the impedance parameters of these components were calculated, and their effects were taken into account in the resonance frequency extraction. $Z_{\text {eq } 1}, Z_{\text {eq } 2}, Z_{\text {eq3 }}$, and $Z_{\text {eq } 4}$ are the impedances that are seen at the input of cable, cable-connector, cable-connector-antenna, and cable-connectorantenna-sensor, respectively.

The inductive coupling between the sensor and the reader antenna affects the impedance at the input of the antenna. Since the sensor impedance $Z_{s}$ reaches a minimum at its resonance frequency $\left(f_{0}\right)$, this provides maximum impedance, which appears at the input of the antenna at $f_{0}$. By scanning the variation on the equivalent impedance, monitored by the reader antenna, the resonance frequency can be extracted. Assuming an inductive coupling between the sensor and reader antenna, the sensor impedance $Z_{s}$, measured by the reader antenna is given by

$V=\left\{\left(R_{1}+j w L_{1}\right)+\left[\left(R_{c}+\frac{1}{j w C_{c}}\right)\left(R_{a}+j w L_{a}-\frac{j w M I_{3}}{I_{2}}\right)\right]\right\} I_{1}$

where $M$ is the mutual coupling coefficient between the sensor and the pick-up antenna,

$\left(R_{s}+j w L_{s}+\frac{1}{j w C_{s}}\right) I_{3}=j w M I_{2}$ 
$R_{s}+j w L_{s}+\frac{1}{j w C_{s}}=Z_{S}$

From (1) and (3);

$Z_{e q}=\frac{V}{I_{1}}=\left(R_{1}+j w L_{1}\right)+\left[\left(R_{c}+\frac{1}{j w C_{c}}\right)\left(R_{a}+j w L_{a}+\frac{w^{2} M^{2}}{Z_{s}}\right)\right](4)$

$Z_{s}=\frac{w^{2} M^{2}\left(R_{1}+j w L_{1}+R_{c}+\frac{1}{j w C_{c}}-Z_{e q}\right)}{\left(Z_{e q}-R_{1}-j w L_{1}\right)\left(R_{c}+\frac{1}{j w C_{c}}+R_{a}+j w L_{a}\right)-\left(R_{c}+\frac{1}{j w C_{c}}\right)\left(R_{a}+j w L_{a}\right)}$

The resonance frequency of the sensor is determined by scanning the frequency response of the sensor impedance, $Z_{s}$. When the sensor stays in the interrogation region of the pick-up reader antenna, a net resonance comes along at the operating frequency of the sensor. The variation of the coupling value would lead to a change in the impedance $Z_{s}$ and, consequently, the shift of the system resonance frequency. Any strain variations on the sensor can be faithfully detected using the associated electrical characteristics ( $f_{0}$ and $\left|\boldsymbol{Z}_{\boldsymbol{s}}\right|$ ) changes, which are picked up by the reader antenna. Each $\left(f_{0}\right.$ and $\left.\left|\boldsymbol{Z}_{\boldsymbol{s}}\right|\right)$ set point is devoted to an individual strain value. Thus, using both frequency and impedance information together strain can be calculated independent of the coupling value.

Here, note that the proposed sensor has two main elements: (1) the split ring capacitors between the fingers, which serve as the sensing component, and (2) the split rings inductor, which collects electromagnetic energy from antenna.

\subsection{Modeling}

The design of the strain sensor relies on the resonance characteristics, especially the resonance frequency, $\mathrm{f}_{0}$ and the quality-factor, $\mathrm{Q}$ which are functions of the sensor capacitance $\left(C_{S}\right)$, inductance $\left(L_{s}\right)$, and resistance $\left(R_{S}\right)$. A simple geometry of the proposed strain sensor with seven geometrical variables is shown in Fig. 2a. The values of $C_{s}, L_{s}$, and $R_{s}$ are determined by these design parameters: number of fingers $\boldsymbol{n}$, gap width $\boldsymbol{s}$, fingers spacing $\boldsymbol{t}$, sensor length $\boldsymbol{k}$, line width $\boldsymbol{w}$, short fingers length $\boldsymbol{l}_{\mathbf{1}}$, and long figures length $\boldsymbol{l}_{\mathbf{2}}$.

The sensor is modeled to operate as an electrical LC resonant circuit. Fig. 2b shows a 3-dimensional (3D) sketch of our proposed strain sensor. The top/front side of the device includes a layer of comb-shaped SRRs metallization. The bottom/back side of the device is the $90^{\circ}$ rotated version of the top/front side that is patterned on the other face of the dielectric substrate. This architecture brings us two main advantages: first, it allows electromagnetic wave to penetrate through the upper layer and bottom one. Accordingly, with proper polarization, both of the layers can be excited by the same incoming electromagnetic wave. Thus, both layers contribute to the operating frequency and Q-factor of the device. Second, due to symmetric structure of the sensor, strain can be measured in any direction.

The sensor has inductance $L_{s}$ and capacitance $C_{s} . C_{S}$ is the combination of the split (metal-air-metal) and sandwiched (metal-substrate-metal) capacitors that build up from the sensor architecture. The integration of capacitance $C_{s}$ with inductance $L_{s}$ forms a resonance circuit. The corresponding resonance frequency is simply

$f_{0}=\frac{1}{2 \pi \sqrt{L_{S} C_{S}}}$

The Q-factor of the sensor affects the precise wireless identification of the sensor resonance frequency. High Q-factor results in strong inductive coupling and the larger interrogation distance. To increase the power reflected by the sensor, the Q-factor of the sensor is increased by improving the structural parameters of the sensor.
The metal thickness is one of the main parameters that can control the Q-factor, consequently the reflected power. To maximize the reflected power back to the antenna, the metal thickness is set to be two times thicker than the electrical skin depth [16]. The well-known equation to calculate the skin depth is given bellow:

$\delta=\sqrt{\frac{2}{2 \pi f_{0} \sigma \mu_{0} \mu_{R}}}$

where $\sigma$ is the conductivity $\left(\mathrm{m}^{-1} \mho\right), \mu_{0}$ the permeability constant $\left(4 \pi \times 10^{-7} \mathrm{H} / \mathrm{m}\right)$, and $\mu_{R}$ the relative permeability. For the target operating frequency of our proposed strain sensor $(\sim 600 \mathrm{MHz})$, the skin depth of gold is calculated to be $\sim 3 \mu \mathrm{m}$. The investigations of the gold skin effect on the Q-factor at the operating frequency shown that the Q-factor is increased by the increasing metal thickness, and becomes fixed after $\sim 6 \mu \mathrm{m}$ metal thickness. Thus, in this study, the gold thickness of $6 \mu \mathrm{m}$ was used in the sensor fabrication.

During the operation, when the sensor is subjected to strain $\epsilon$ in each direction, the geometrical deformation on the sensor leads to change in the split ring capacitance (between fingers) values, which results in the resonance frequency shift. The variation of capacitance values contribute to the resonance frequency shift and, consequently strain sensing.

\subsection{Device design and fabrication}

Our proposed architecture is based on a multilayer laminated structure consisting of two comb-shaped SRRs, which are patterned on both sides of the dielectric substrate, with $90^{\circ}$ alignment with respect to each other (Fig. 2). We fabricated our strain sensor using standard microfabrication technique. The fabrication processes include the following steps: (1) Thermal evaporation was used to deposit a thin layer of Au on both sides of the flexible dielectric substrate (Kapton ${ }^{\circledR}$ polyimide films, DuPont ${ }^{\mathrm{TM}}$ ). (2) Conventional lithography and wet-etching were used to pattern the Au layers of comb-shaped SRRs on both sides of 25- $\mu$ m-thick Kapton. Finally, a flexible sensor with the thickness of $\sim 37 \mu \mathrm{m}$ was obtained. A photo of the fabricated sensor on the flexible Kapton film and the corresponding optical image are displayed in Fig. 2c, along with all physical dimensions. Due to the flexibility and ultrathin structure of the sensor, the sensor has the capability to conform to various non-planar surfaces.

\subsection{Experimental setup and measurements}

Our experimental setup and corresponding block diagram utilized for the telemetric strain monitoring is shown in Fig. 3. The fabricated sensor is set on the host structure to characterize the sensor performance in strain measuring. The host structure is a homo-polymer rod; namely, Delrin ${ }^{\circledR}$ with dimensions $1.2 \mathrm{~cm} \times 1.2 \mathrm{~cm} \times 10.0 \mathrm{~cm}$ and Young's modulus of $2.4 \mathrm{Gpa}$. The axial tensile force of $2 \mathrm{kN}$ was applied to the system in four loading steps with $500 \mathrm{~N}$ load increment per step using a tensile tester machine (INSTRONTM 5542). To verify the uniform strain induction in the area where the sensor is mounted, a standard resistive strain gauge (Tokyo Sokki Kenkujo Co., Ltd.) is set to the host structure as shown in Fig. 3. The strain gauge is positioned on the other side of the Delrin, which does not affect the sensor performance. The test starts with $0 \mu \varepsilon$ initial load and ends at around 21,300 $\mu \varepsilon$. The impedance of the strain sensor is measured at the various interrogation distances using the pick-up coil (with $8 \mathrm{~mm}$ in diameter) as the reader antenna under each loading step.

The reader antenna is mounted on a 3D stage machine (VELMEX, Inc.). The stage automatically interrogates the region step by step. We used a network analyzer (Agilent FieldFox N9915A) as the signal acquisition instrument that was arranged in the operating 

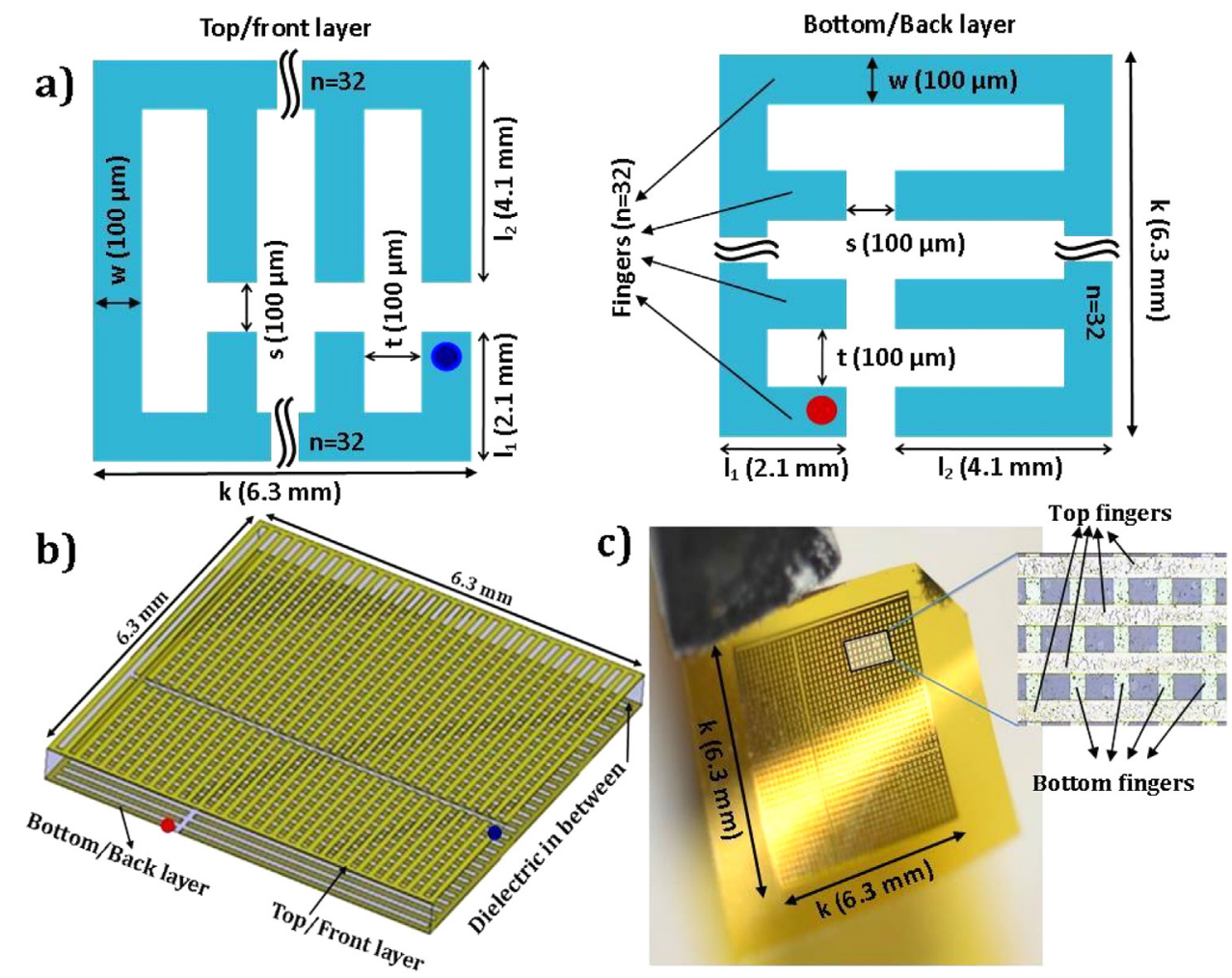

Bottom fingers

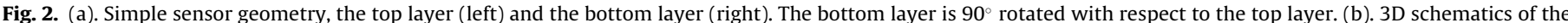

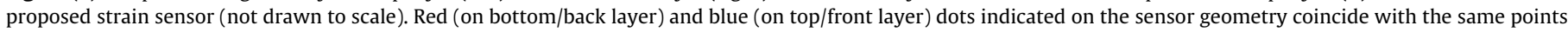

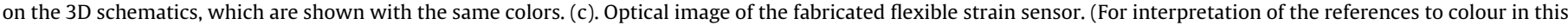
figure legend, the reader is referred to the web version of this article.)

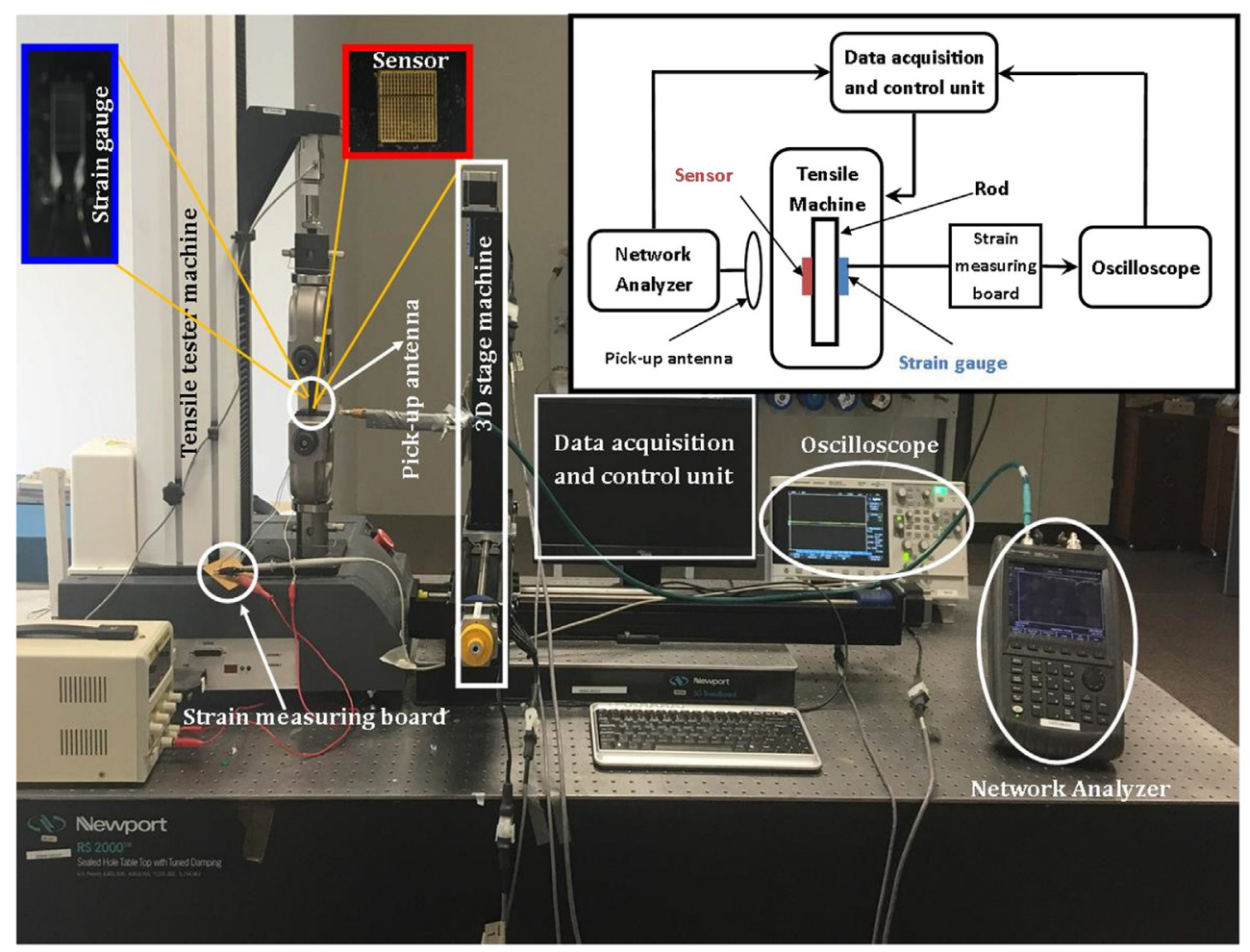

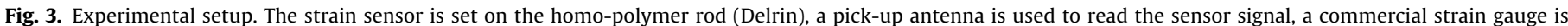

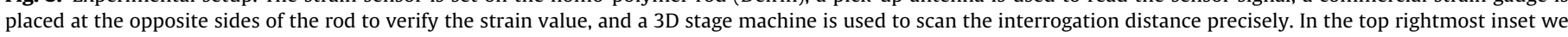

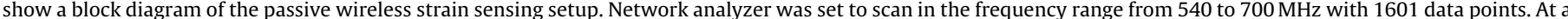

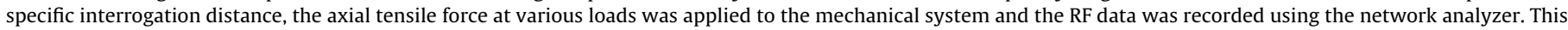
was repeated at varied interrogation distances. 


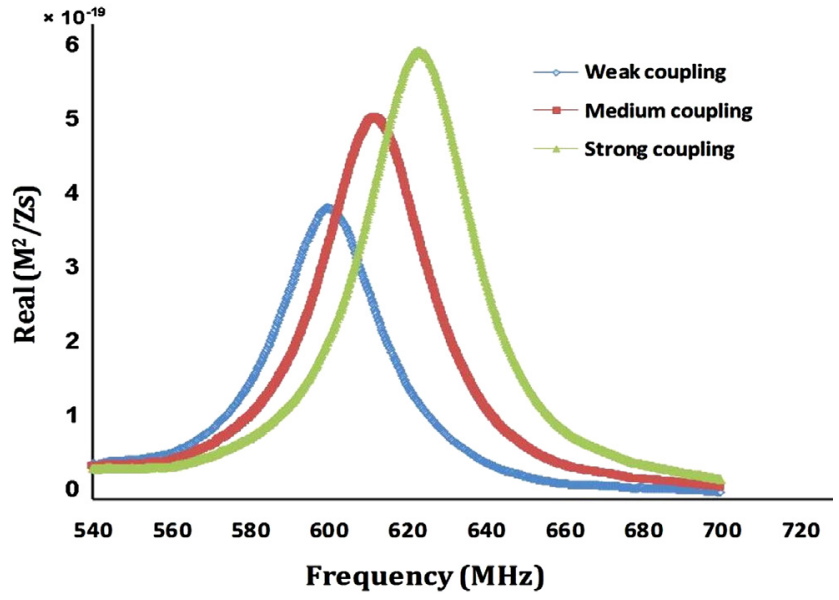

Fig. 4. By changing the inductive coupling (by varying the interrogation distance) between the reader antenna and sensor, the operating frequency of the system and impedance of the system $\left(\mathrm{Z}_{\text {eq } 4}\right)$ were changed. The strong coupling, medium coupling, and weak coupling correspond to the distances of $0.5 \mathrm{~mm}, 1.3 \mathrm{~mm}$ and $2.4 \mathrm{~mm}$ far from the pick-up antenna, respectively.

frequency range with 1601 data points. The experiment was conducted at ten interrogation distances from 0.5 to $3.0 \mathrm{~mm}$. The sensor with following design parameters was used in the strain measuring experiment: $\mathrm{n}=32, \mathrm{~s}=100 \mu \mathrm{m}, \mathrm{t}=100 \mu \mathrm{m}, \mathrm{k}=6.3 \mathrm{~mm}, \mathrm{w}=100 \mu \mathrm{m}$, $\mathrm{l}_{1}=2.1 \mathrm{~mm}$, and $\mathrm{l}_{2}=4.1 \mathrm{~mm}$.

\section{Results and discussion}

Before studying the strain sensor performance, the effect of interrogation distance was investigated. As it has been expected, changing the interrogation distance the level of inductive coupling between the sensor and the pick-up antenna varies, as shown in Fig. 4. Due to the coupling coefficient variation, the system operating frequency changes at various interrogation distances. The variation in the operating frequency can be larger than the shifts caused by the applied load. Therefore, it becomes impossible to attribute an individual resonance frequency value to an individual strain value, consequently resulting in discrepancies in the strain extraction, unless specific calibration curves are used at specific fixed interrogation distances.

To solve this problem, the sensor was tested under different load values and varying interrogation distances to find relation between the strain, $\left|\boldsymbol{Z}_{\boldsymbol{s}}\right|$, and $\mathrm{f}_{0}$. Here both the frequency and $\left|\boldsymbol{Z}_{\boldsymbol{s}}\right|$ information are used to extract the individual strain value. Fig. 5a shows the resonance frequency variation by changing the inductive coupling coefficient (by changing the interrogation distance) under various strain values. This figure also shows a clear resonance frequency increase with the increasing coupling coefficient.

To investigate the tensile strain response of the sensor as the strain increases, the resonance frequency is measured at different strain values. The relation between the resonance frequency shift of the sensor and strain is displayed in Fig. 5. This plot confirms that the measured resonance frequency of the sensor at various load values exhibits a characteristic linear response. The measured resonance frequency of the sensor gradually increases as the strain increases. The resonance frequency of the sensor at $0 \mu \varepsilon$ is around $591 \mathrm{MHz}$, and then it reaches approximately to $597 \mathrm{MHz}$, as the strain increases up to $10,500 \mu \varepsilon$. The experimental results show that the sensor achieves successful strain performance with the high sensitivity. Due to the proximity of the sensor and pick-up antenna, mutual coupling is more dominant, which does not allow for the significant resonance frequency change by the strain variation. Strong coupling between the sensor and the antenna at this a)

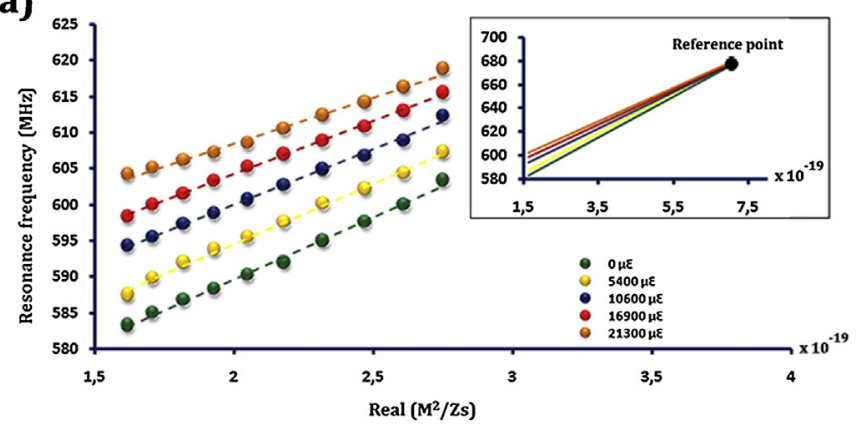

b)

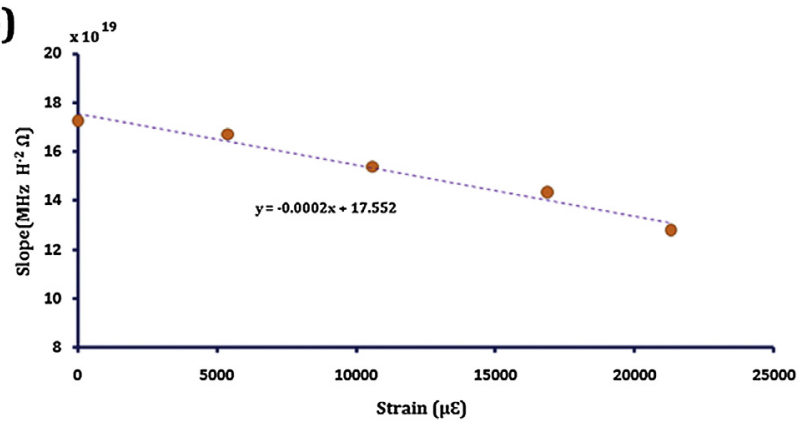

Fig. 5. (a) Resonance frequency variation under different strain levels at different interrogation distances. Linear fits at various strain values intersect at a common interpolated reference point. Due to close proximity of the sensor and reader antenna (dominant coupling effect), measured signals tend to intersect at this point at different strain levels. (b). The calculated slope of any measured point with the reference point corresponds to an individual strain value (measured by strain gauge).

point allows all linear fits at various strain levels (Fig. 5a) tend to a single intersecting point which we call Reference Point here. Fig. 5b shows the slope values of these linear fits at various strain levels, measured by the strain gauge. Any measured set point $\left(f_{0}\right.$ and $\left.\left|\mathbf{Z}_{\mathbf{s}}\right|\right)$ creates a slope by the Reference Point. This slope gives the extracted strain value.

After the strain characterization and analyzing the relationship between the strain and set point $\left(f_{0}\right.$ and $\left.\left|\mathbf{Z}_{\mathbf{s}}\right|\right)$, similar tensile test is conduced to investigate the sensor performance and verification. The sensor was subjected to a random set of strain levels and interrogation distance values. The experimental error analysis in Fig. 6a exhibited excellent sensor performance. The fitted curve in this figure reports a maximum error of only less that $0.5 \%$ corresponding to nonlinearity error at the early stages of the strain implementation. This could be largely explained by the dominant effect of the uncorrelated measured strain between the strain gauge and our sensor. Also, some part of this error stems from the strain gauge voltage measurement error. By increasing the strain, calculated error was minimized, which could be explained by the dominant effect of the applied strain. The resonance frequency and $\left|\boldsymbol{Z}_{\boldsymbol{s}}\right|$ of the sensor can be interrogated by the pick-up antenna in the range of $3 \mathrm{~mm}$. Beyond this distance, due to the very low coupling efficiency, the reader antenna is not be able to sense the signal reflected back from the sensor. Therefore, this distance is the largest operating range for our system.

To investigate the effect of the direction of the applied load on wireless strain measurement, the experiment was conducted at two different sensor orientations. The strain was applied in both $x$ and $y$ directions. Due to symmetric structure of the sensor, the experiments resulted in the same behavior.

The stability and reliability of the sensor were studied by multicycle strain performing on the sensor. The sensor was first subjected to zero strain loading and then followed by extension loading of $5400 \mu \varepsilon$. As shown in Fig. 6b, these steps were repeated 

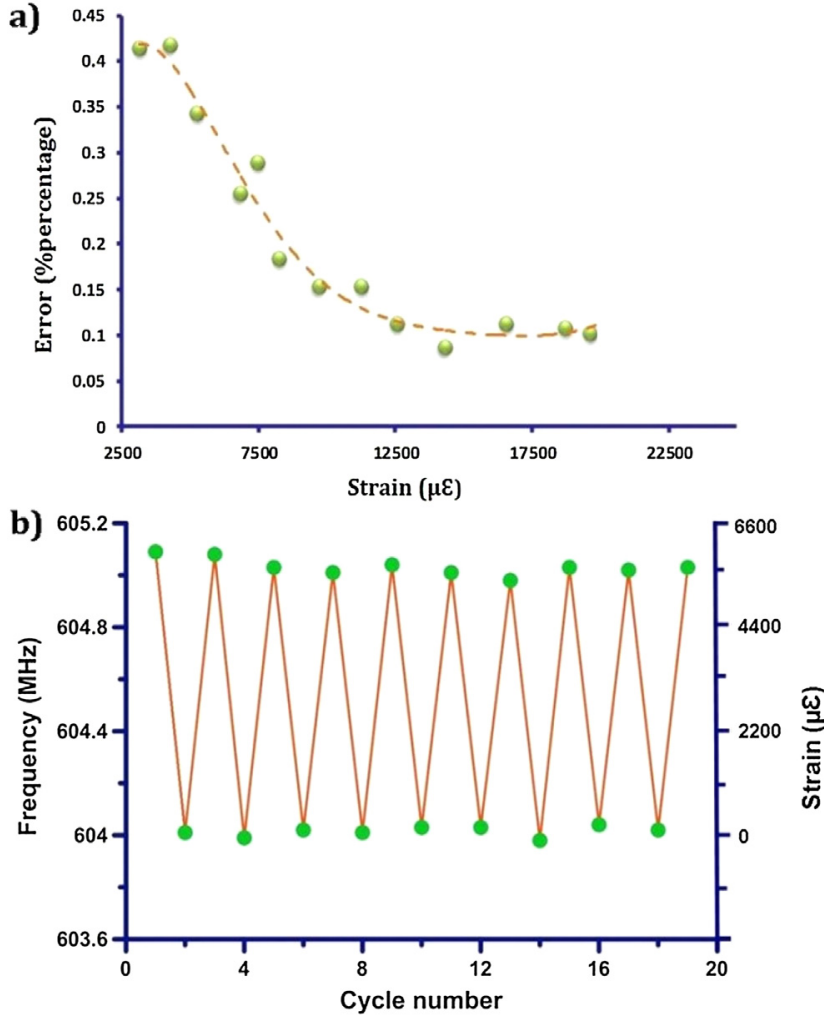

Fig. 6. (a) Nonlinearity error percentage. At high strain values error was minimized. (b). The multicycle operation of the sensor between the two strain states (achieved by strain gauge) showed that the response of the sensor is stable and reliable.

20 times. The plot of the repeatability showed that the sensor showed excellent stability and reliability, with only slight drifts $(<0.01 \%)$ observed between the cycles.

\section{Conclusion}

The present study reports a new telemetric measurement technique and a passive RF sensor for distance-independent wireless strain monitoring. Distance-independent strain monitoring is achieved by measuring the impedance of the sensor in addition to its resonance. In our proposed telemetric monitoring, we include the impedance parameters of all electrical components to measure precisely the resonance frequency of the sensor. The proposed sensor consists of an ultra-thin and multilayer (metal-dielectric-metal) structure with comb-shaped SRRs that patterned on both sides of the flexible dielectric. Top and bottom layers of the sensor are $90^{\circ}$ rotated with respect to each other. The specific architecture of this design allows for the both metal layers to contribute in the sensor electrical characteristics $\left(f_{0}, Q\right.$-factor, and $\left.Z_{s}\right)$. The sensor is tested under various strain values and interrogation distances. A linear relation between the sensor $f_{0}$ and $\left|Z_{\boldsymbol{s}}\right|$ is obtained at different levels of strain. The slope of this response gives the strain. This strong linearity results in excellent strain sensing performance. By taking the advantages of this telemetric measurement technique and sensor structure, distance-independent strain extraction has been successfully demonstrated in this study. The results present that the sensor has excellent accuracy with less than $0.5 \%$ error, and also showed great stability and reliability.

Our future study will focus on the application areas of this strain sensor. The proposed sensor has great potential for performing faithful wireless strain measurement in vivo while the patient's body parts may be moving. This can be used to register strain variation precisely during bone fracture healing even when the patient fracture site cannot be precisely located externally. Furthermore, future research needs to be conducted to improve sensing performance at longer interrogation distances. These findings given for telemetric strain monitoring may be a promising wireless strain measurement method in the systems with varying interrogation distance. Studies performed in passive wireless monitoring showed that changing the interrogation distance may result in inaccurate measurement. The proposed approach here resolves the problem of interrogation distance variation.

\section{References}

[1] P. Zhao, N. Deng, X. Li, C. Ren, Z. Wang, Development of highly-sensitive and ultra-thin silicon stress sensor chips for wearable biomedical applications, Sens. Actuators A Phys. 216 (2014) 158-166, http://dx.doi.org/10.1016/j.sna. 2014.05.018.

[2] P.J. A, S.F. Pichorim, Biotelemetric passive sensor injected within tendon for strain and elasticity measurement, IEEE Trans. Biomed. Eng. 53 (2006) 921-925, http://dx.doi.org/10.1109/TBME. 22006.872157.

[3] E.L. Tan, W.N. Ng, R. Shao, B.D. Pereles, K.G. Ong, A wireless, passive sensor for quantifying packaged food quality, Sensors 7 (2007) 1747-1756, http://dx. doi.org/10.3390/s7091747.

[4] John C. Butler, JAnthony J. Vigliotti, Fred W. Verdi, Shawn M Walsh, Wireless, passive, resonant-circuit, inductively coupled, inductive strain sensor, Sensor Actuat. A: Phys. 102 (2002) 61-66, http://dx.doi.org/10.1016/S09244247(02)00342-4.

[5] P. Broutas, H. Contopanagos, E.D. Kyriakis-Bitzaros, D. Tsoukalas, S. Chatzandroulis, A low power RF harvester for a smart passive sensor tag with integrated antenna, Sensor Actuat. A: Phys. 176 (2012) 34-45, http://dx.doi. org/10.1016/j.sna.2011.12.053.

[6] Y. Jia, K. Sun, F.J. Agosto, M.T. Quiñones, Design and characterization of a passive wireless strain sensor, Meas. Sci. Technol. 17 (2006) 2869, http://dx. doi.org/10.1088/0957-0233/17/11/002.

[7] Hee-Jo Lee, Jong-Gwan Yook, Recent research trends of radio-frequency biosensors for biomolecular detection, Biosens. Bioelectron. 61 (2014) 448-459, http://dx.doi.org/10.1016/j.bios.2014.05.025.

[8] William B. Spillman Jr., S. Durkee, Noncontact power/interrogation system for smart structures, SPIE 2191 (1994) 362-372, http://dx.doi.org/10.1117/12. 173966.

[9] X. Yi, C. Cho, J. Cooper, Y. Wang, M.M. Tentzeris, R.T. Leon, Passive wireless antenna sensor for strain and crack sensing-electromagnetic modeling, simulation, and testing, Smart Mater. Struct. 22 (2013) 085009, http://dx.doi. org/10.1088/0964-1726/22/8/085009.

[10] A. Daliri, A. Galehdar, W.S.T. Rowe, K. Ghorbani, S. John, Utilising microstrip patch antenna strain sensors for structural health monitoring, Intell. Mater. Syst. Struct. 23 (2011) 169-182, http://dx.doi.org/10.1177/ $1045389 \times 11432655$.

[11] A. Daliri, A. Galehdar, S. John, C.H. Wang, W.S.T. Rowe, K. Ghorbani, Wireless strain measurement using circular microstrip patch antennas, Sensor Actuat. A Phys. 184 (2012) 86-92, http://dx.doi.org/10.1088/0964-1726/22/8/085009.

[12] X. Yi, T. Wu, Y. Wang, R.T. Leon, M.M. Tentzeris, G. Lantz, Passive wireless smart-skin sensor using RFID-based folded patch antennas, Int. J. Smart Nano Mater. 2 (2011) 22-38, http://dx.doi.org/10.1080/19475411.2010.545450.

[13] E.U.R. Melik, N.K. Perkgoz, B. Santoni, D. Kamstock, C. Puttlitz, H.V. Demir, Nested metamaterials for wireless strain sensing, IEEE J. Sel. Top. Quantum Electron. 16 (2010) 450-458, http://dx.doi.org/10.1109/JSTQE.2009.2033391.

[14] K.C. McGilvray, E. Unal, K.L. Troyer, B.G. Santoni, R.H. Palmer, J.T. Easley, H.V. Demir, C.M. Puttlitz, Implantable microelectromechanical sensors for diagnostic monitoring and post-surgical prediction of bone fracture healing, J. Orthop. Res. 33 (2015) 1439-1446, http://dx.doi.org/10.1002/jor.22918.

[15] I. Jones, L. Ricciardi1, L. Hall, H. Hansen, V. Varadan, C. Bertram, S. Maddocks, S. Enderling, D. Saint, S. Al-Saraw, Wireless RF communication in biomedical applications, Smart Mater. Struct. 17 (2008) 015050, http://dx.doi.org/10. $1063 / 1.3250175$.

[16] D.A. Sanz, C. Mitrosbaras, E.A. Unigarro, F. Segura-Quijanod, Passive resonators for wireless passive sensor readout enhancement, Appl. Phys. Lett. 103 (2013) 133502, http://dx.doi.org/10.1088/0964-1726/17/1/015050.

\section{Biographies}

Akbar Alipour received the B.Sc. in Electrical and Electronics Engineering from Urmia University, Urmia, Iran in 2007. He completed his Master degree in biomedical science at METU and Gazi University, Ankara, Turkey in 2011. Since 2012, he is working towards the Ph.D. degree with the department of Electrical and Electronics Engineering, Bilkent University. His current research interests include the implant sensors for biomedical applications.

Emre Unal received his B.S. degree in electrical and electronics engineering from Hacettepe University, Ankara, Turkey, in 2005. He is a full-time Research Engineer under the supervision of Prof. H. V. Demir with the Institute of Materials Science 
and Nanotechnology, Bilkent University, Ankara, where he is working on the development of microwave and optoelectronic devices.

Sayim Gokyar received his B.S. degree in electrical and electronics engineering from Fatih University, Istanbul, Turkey, in 2009. He completed his M.S. degree in electrical and electronics engineering from Bilkent University, Ankara, Turkey, in 2011. He is currently a $\mathrm{PhD}$ candidate at electrical and electronics engineering department, Bilkent University, Ankara, Turkey. His research area of interest includes wireless sensors.

Hilmi Volkan Demir (M'04-SM'11) received the B.S. degree in electrical and electronics engineering from Bilkent University, Ankara, Turkey, in 1998 and the M.S. and $\mathrm{Ph} . \mathrm{D}$. degrees in electrical engineering from Stanford University, Stanford, CA, in 2000 and 2004, respectively. In September 2004, he joined Bilkent University, where he is currently a professor with joint appointments at the Department of Electrical and Electronics Engineering and the Department of Physics and is also with the Institute of Materials Science and Nanotechnology. Concurrently, he is a fellow of National Research Foundation in Singapore and a professor of Nanyang Technological University. His research interests include the development of innovative optoelectronic and RF devices. Dr. Demir was the recipient of the European Union Marie Curie Fellowship, the Turkish National Academy of Sciences Distinguished Young Scientist Award (TUBA-GEBIP), the European Science Foundation-European Young Investigator Award (ESF-EURYI), and Nanyang Award for Research Excellence. 